\title{
Size dependence of solar X-ray flare properties
}

\author{
M. Battaglia, P. C. Grigis, and A. O. Benz
}

Institute of Astronomy, ETH Zentrum SEC, 8092 Zürich, Switzerland

e-mail: battaglia@astro.phys.ethz.ch

Received 8 March 2005 / Accepted 24 April 2005

\begin{abstract}
Non-thermal and thermal parameters of 85 solar flares of GOES class B1 to M6 (background subtracted classes A1 to M6) have been compared to each other. The hard X-ray flux has been measured by RHESSI and a spectral fitting provided flux and spectral index of the non-thermal emission, as well as temperature and emission measure of the thermal emission. The soft X-ray flux was taken from GOES measurements. We find a linear correlation in a double logarithmic plot between the nonthermal flux and the spectral index. The higher the acceleration rate of a flare, the harder the non-thermal electron distribution. The relation is similar to the one found by a comparison of the same parameters from several sub-peaks of a single flare. Thus small flares behave like small subpeaks of large flares. Thermal flare properties such as temperature, emission measure and the soft X-ray flux also correlate with peak non-thermal flux. A large non-thermal peak flux entails an enhancement in both thermal parameters. The relation between spectral index and the non-thermal flux is an intrinsic feature of the particle acceleration process, depending on flare size. This property affects the reported frequency distribution of flare energies.
\end{abstract}

Key words. Sun: flares - Sun: X-rays, $\gamma$-rays - acceleration of particles

\section{Introduction}

Do small flares differ from large flares? In the simplest flare scenarios small flares are just scaled down versions of large flares. This is predicted explicitly in theories that envision flares as a kind of avalanche process, consisting of many small elements (Lu \& Hamilton 1991; Lu et al. 1993). On the other hand, one could speculate that a flare changes the environment of an active region and, in its course, alters the properties of the elementary events. Such an effect would be a feedback effect, thus a secondary phenomenon. However, big flares could be genuinely different because the initial conditions that lead to them may be more demanding on some plasma parameters that finally not only make them bigger but qualitatively dissimilar.

Kahler (1982a) noted that there is a statistical correlation between solar flare energy release and the observation of certain flare manifestations. This so-called "Big Flare Syndrome" states that large flares tend to be associated with phenomena that may not be directly related to each other, such as solar protons (Kahler 1982b), type II and IV radio bursts (Kahler 1982b), decimeter radio emissions (Simnett \& Benz 1986), coronal mass ejections (Dougherty et al. 2002), and white light flares (Matthews et al. 2003). A simple interpretation of the Big Flare Syndrome proposes that the various signatures just get above the threshold for observations in large events (Kahler 1982a). However, some radiations do not increase linearly with flare size. Such a behavior was e.g. recently reported for the energy in decimeter type IV bursts, increasing with about the fifth power of the GOES soft X-ray flux (Benz et al. 2005).

A quantitative difference between small and large flares was found in thermal soft X-ray emission. Feldman et al. (1996) report an increase of temperature $T$ with the flare soft X-ray emission, defined by the GOES peak flux $F_{\mathrm{G}}$ at $1-8 \AA$ $(1.55-12.4 \mathrm{keV})$. The average relation is approximately

$F_{\mathrm{G}}(T)=3.5 \times 10^{0.185 T-9.0}$,

where the flux is in $\mathrm{W} \mathrm{m}^{-2}$ and the temperature in units of $10^{6} \mathrm{~K}$. They also find a correlation between emission measure and temperature which has been explained theoretically by Shibata \& Yokoyama (1999) based on a balance between magnetic reconnection and conductive cooling. Feldman et al. conclude that if a flare is the collection of subresolution events, the plasma properties of the events occurring during peak emission of large flares and small flares must be different.

Here we ask whether the characteristics of hard X-rays emitted by the non-thermal electrons accelerated in solar flares are different in small and large flares. Except for the rare thermal flares, the energy distribution of hard X-ray flare photons follows a power-law. Its index $\gamma$ usually starts with a high value ("soft" radiation), reaches a minimum at peak flux and increases at the end (as noticed already by Parks \& Winckler 1969). This "soft-hard-soft" (SHS) behavior is also found in sub-peaks and has recently been analyzed quantitatively for the first time by Grigis \& Benz (2004, henceforth GB04) using RHESSI observations. The SHS evolution of individual flares 
and subpeaks indicates a change in the acceleration process in the course of a flare. It can be visualized roughly by the nonthermal spectrum in log-log representation moving down and up at high energies with a fixed point in the flux-index plane, at an energy of 6.5-12.5 keV (average $9 \mathrm{keV}$; GB04). Although this "pivot" point may be virtual (i.e. an extrapolation beyond measured data), its location is a characteristic of the electron acceleration process and the subsequent diffusion in energy.

For all rise and decay phases in several subpeaks of 24 flares, GB04 find the relation

$\gamma=A F_{35}^{-\alpha}$,

where $F_{35}$ is the fitted non-thermal flux at $35 \mathrm{keV}$, and $\alpha=$ $0.197 \pm 0.003$. Does the same relation hold between the flux and spectral index at peak time for flares of different size? Another question is whether the flares of different size behave in the average like the subpeaks of one flare. X-ray data of a large number of flares, small and large, have been registered by the Ramaty High Energy Solar Spectroscopic Imager (RHESSI, Lin et al. 2002). Section 2 describes how an equal number of flares in each decade of peak GOES flux was selected. In Sect. 3, the characteristic values of thermal and nonthermal emissions at peak flux are compared as a function of flare size. The results are discussed and compared to previous work in Sect. 4, and conclusions drawn in Sect. 5.

\section{Event selection and data reduction}

The RHESSI satellite observes the full Sun from $3 \mathrm{keV}$ to $17 \mathrm{MeV}$ since February 2002. In this study, the high spectral resolution $(1 \mathrm{keV})$ of RHESSI's germanium detectors is utilized to study the hard X-ray flare emission below $300 \mathrm{keV}$. This energy range is dominated by a thermal part at the lower end, usually superposed by a non-thermal part with a powerlaw spectrum at higher energies. The high spectral resolution allows for the first time to separate and to analyze the nonthermal component where it comprises most photons in a large number of flares. The same analysis also yields information on the thermal X-ray emission in the $3-20 \mathrm{keV}$ range. It is supplemented by soft X-ray observations from the GOES satellite (Garcia 1994).

We selected 100 well observed flares from GOES class B1 to X1 from the time between RHESSI launch and April 5th 2004, using the RHESSI Experimental Data Center (HEDC, Saint-Hilaire et al. 2002) at ETH Zürich. The selection process aimed at having a statistically representative sample of flares over the whole time period and from different active regions.

\subsection{Data selection}

We took all events with an image in HEDC, giving their location on the solar disc, and present in the RHESSI-flare-list of April 5th, 2004. This ensured that only solar events were selected and yielded a list of 6039 flares. As we wanted to arrange the flares according to their GOES class, flares without or with bad GOES data in the corresponding time interval where removed from the list. This second step ended with a list of 5871 flares.

To get a uniform distribution of events in flare size, flares where sorted according to their GOES peak flux (without background subtraction). The flux range $\mathrm{B} 1$ to $\mathrm{X} 1$ was divided into 10 equally wide bins on a logarithmic scale. For each of the 10 bins, 10 events where randomly chosen. A flare had to fulfill the following criteria to be acceptable:

1. The start of the flare and its peak hard X-ray emission had to be well observed. This should guarantee that we did not miss any interesting parts and allow a good background selection.

2. The attenuation state had to be constant for a period of 4 min centered at hard X-ray peak time.

3. The front decimation weight had to be less or equal to 2 , to better study the thermal emission. There was an exception from this rule for GOES classes M5 to X1, because in this bin there were just 8 events. Thus every single flare that fulfilled the first 2 criteria has been taken to have a sample as large as possible.

4. For flares in GOES class B1 to B9, there had to be no attenuation and decimation, because of the weak emission of these flares and the low count rates.

5. There must be no enhanced flux of charged particles in the satellite environment a few minutes before and after peak time.

We additionally discarded small flares occurring during the decay phase of a larger event since in such cases it is not possible to separate the emissions of the two events.

For each of the selected flares we performed a spectral analysis. We chose the longest time interval around flare peak time without gaps and with RHESSI in sunlight, and we created a series of spectra with a time bin with of one RHESSI rotation period (approximately $4 \mathrm{~s}$ ). The energy-bin width was chosen fine enough to resolve the thermal and non-thermal spectra, but not so fine that statistical errors became too large. In our case that meant a binning of $1 \mathrm{keV}$ from $3-50 \mathrm{keV}$ (with exception of the range $6-12 \mathrm{keV}$ for the larger flares (C3-X1), where the binning was $0.3 \mathrm{keV}$ ), and a larger binning above $50 \mathrm{keV}$. With these bin widths, a clear separation between the thermal and non-thermal spectrum is possible. For smaller flares, a wider binning was applied for energies of $20 \mathrm{keV}$ and higher as there is less flare emission above that energy. Only the data from the front segments, without detectors 2 and 7, where used (in case the transmitter was active during the chosen time interval, detector 8 was also omitted).

For 7 events, no spectrum files could be produced. One event had the lifetime below $90 \%$ at peak time and therefore was not acceptable for spectroscopy. This left us with 92 events.

\subsubsection{Selection effect for small flares}

The RHESSI flare list reports events automatically flagged by the software when the emission in the $12-25 \mathrm{keV}$ band increases. This energy range contains some non-thermal emission of microflares (Benz \& Grigis 2002; Krucker et al. 2002). 


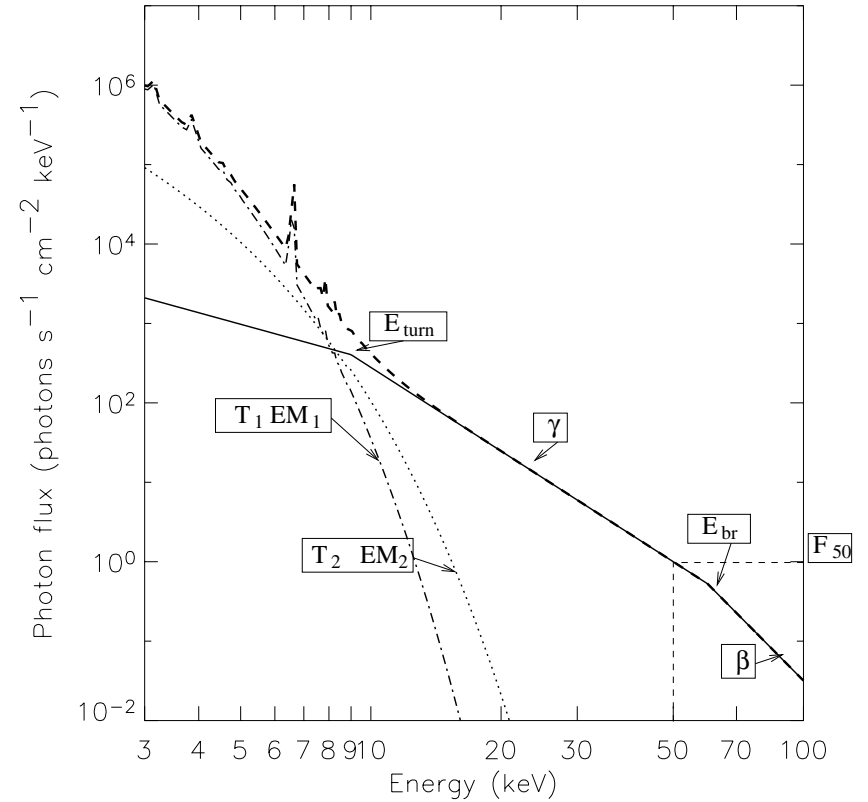

Fig. 1. Illustration of the fitting model and the 9 free parameters discussed in the text. A non-thermal component (solid line) and two thermal components (dotted and dash-dotted) are shown.

However, weak GOES events tend to be lost in the RHESSI background in this energy range. Therefore, the RHESSI flare list misses many soft X-ray events of lower GOES class. We have tried to compensate for it by choosing manually 14 flares from the observing summary light curves that did not have a flare flag. For all of those events, the non-thermal emission was however too small to perform a spectral fitting in such a way that the event could be used meaningfully in the further analysis. Thus there remains a selection effect: for low GOES classes, events with large 12-25 keV flux are preferentially selected. Comparisons between non-thermal emission and thermal emission (GOES class), where this effect may play a role, must be treated with special attention. We will point them out in the discussion (Sect. 4). The influence of the selection effect on the relation between the non-thermal parameters is shown in Fig. 2.

\subsection{Background subtraction and peak time selection}

For each event we subtracted the background and performed a spectral fitting at the time of maximum emission in the hardest observable peak. As the spectral index changes with time, we chose a fitting time-interval of only $4 \mathrm{~s}$ to have a value for the instantaneous spectral index, rather than one averaged over time. The fitting has been performed using the SPEX package (Schwartz 1996; Smith et al. 2002). In total, 3 time intervals have been fitted: one at peak time, and one immediately before and after the peak time interval to compare the fittings and see whether they were plausible. For the later analysis only the spectrum at peak time has been used.

\subsection{Spectral fitting}

SPEX transforms a model photon spectrum into a model count spectrum via the spectral response matrix (SRM) and compares it to the observed count spectrum, iteratively adjusting the model parameters until a local minimum in $\chi^{2}$ is found.

We used a spectral model that consisted of 2 isothermal components, each given by its temperature $T_{1}$ and $T_{2}$ as well as its emission measure $E M_{1}$ and $E M_{2}$ respectively, and a nonthermal component. The non-thermal component consists of a power-law with spectral index $\gamma$ and flux $F_{50}$ at normalization energy $50 \mathrm{keV}$, a low energy turnover at energy $E_{\text {turn }}$ and a highenergy break at energy $E_{\mathrm{br}}$. The power-law index above $E_{\mathrm{br}}$ is named $\beta$. The index below $E_{\text {turn }}$ is fixed at 1.5 . The model is illustrated in Fig. 1. It has 9 free parameters, but it was not always necessary to let all of them free. At first, an isothermal component $\left(T_{1}, E M_{1}\right)$ was fitted, followed by a powerlaw with a low-energy turnover (henceforth called "standard model"). If the non-thermal spectrum showed indications of a break at high energies, the parameters $E_{\mathrm{br}}$ and $\beta$ were also fitted. Some of the larger flares showed strong thermal emission that was fitted with an additional hotter isothermal component $\left(T_{2}, E M_{2}\right)$.

All 92 flares are listed in Table 1 with a number, date, peak time, GOES flux, spectral index and non-thermal flux. In total, 6 different models where fitted:

1. One thermal part and an unbroken power-law (standard model): this provided the best fitting for 31 flares. Another 37 flares where fitted with this model, but were also consistent with two thermal components and no power-law. This point will be discussed later.

2. One thermal part and a broken power-law: for 15 of the larger flares, a broken power-law fitting was applied. Most of these flares had emission beyond $50 \mathrm{keV}$, some up to $100 \mathrm{keV}$ or more. Three of them can also be fitted with two thermal components.

3. Two thermal parts plus power-law: 2 flares had thermal emission up to around $20 \mathrm{keV}$ which we fitted with two temperatures instead of one.

4. Only thermal: 6 flares show no indications for a power-law part in the spectrum and were therefore fitted with one thermal part only (flare numbers 86-90 in Table 1) and with only two thermal components (No. 91). The largest of them was a C1.3 after background subtraction. These flares were not used in the further analysis.

5. Only broken power-law: flare No. 92 (Table 1) was particularly difficult to fit. The best fitting was a broken powerlaw only. The spectrogram shows a very impulsive, short emission, even at the lowest energies. If present at all, the thermal emission must have been very small. This flare was not taken into the further analysis.

In conclusion, 85 out of 92 events could be fitted with the standard model or additionally with a break or a second isothermal component. 


\subsection{Flares with more than one fitting model}

For many of the flares smaller than background subtracted GOES class $\mathrm{C} 2$, fitting a model with only 2 thermal components and no non-thermal emission was also possible. These smaller flares are less intensive than large flares. Therefore the non-thermal emission can be quite weak or even lie below the background level. As the signal-to-noise ratio decreases, different fittings are possible, but not necessarily plausible. The $\chi^{2}$ of the two different models are often about the same value, making it impossible to determine which model is better. The temperatures range up to $260 \mathrm{MK}$ in B-class flares. Although the thermal energy content would not be unphysically high, the standard model has been taken for each of those flares in the further analysis, as the analogy to large flares is the simplest assumption.

\section{Results}

We present here the results obtained from the fittings, comparing several flare properties to each other.

\subsection{Non-thermal emission}

\subsubsection{Spectral index versus non-thermal photon flux}

The non-thermal photon flux is modeled by a power-law distribution with spectral index $\gamma$. For events with a broken powerlaw, $\gamma$ refers to the spectral index below the break energy. For the comparison of the spectral index and the photon flux we cannot use the total flux above $E_{\text {turn }}, F_{\text {tot }}=\int_{E_{\text {turn. }}}^{\infty} F(E) \mathrm{d} E$, since this quantity depends strongly on $E_{\text {turn }}$ which is poorly determined by the observations. Instead we use the flux at a reference energy $E_{0}$. The maximum energy with still observable flare emission varies from about $20 \mathrm{keV}$ to $300 \mathrm{keV}$, and increases with flare size. Therefore, choosing a high $E_{0}$ means extrapolating the flux of small flares into a range where it is not actually observed, whereas choosing a low $E_{0}$ means extrapolating the flux of large flares into a range where thermal emission might dominate. The influence of the choice of $E_{0}$ on the plot of $\gamma$ vs. $F_{E_{0}}$ is described in GB04. We chose $E_{0}=$ $35 \mathrm{keV}$ as a typical energy for the presentation of the results. This allows also for comparisons with previous work.

The plot of $\gamma$ vs. $F_{35}$ for the examined 85 flares with a nonthermal component is shown in Fig. 2. Despite the large scatter of the data, a linear correlation in the double logarithmic plot is noticeable. A linear regression has been performed. As suggested by Isobe et al. (1990) for this kind of scattered data, the bisector regression method has been used. The relation can be written as a power-law model

$\ln \gamma=\ln (A)-\alpha \ln F_{35}$,

with statistical errors $\ln (A)=1.11 \pm 0.06$ and $\alpha=0.13 \pm$ 0.01 , or

$\gamma=3.0 F_{35}^{-0.13}$.

Since the data have a fairly large scatter and there are less than 100 data points, it is difficult to determine unambiguously a

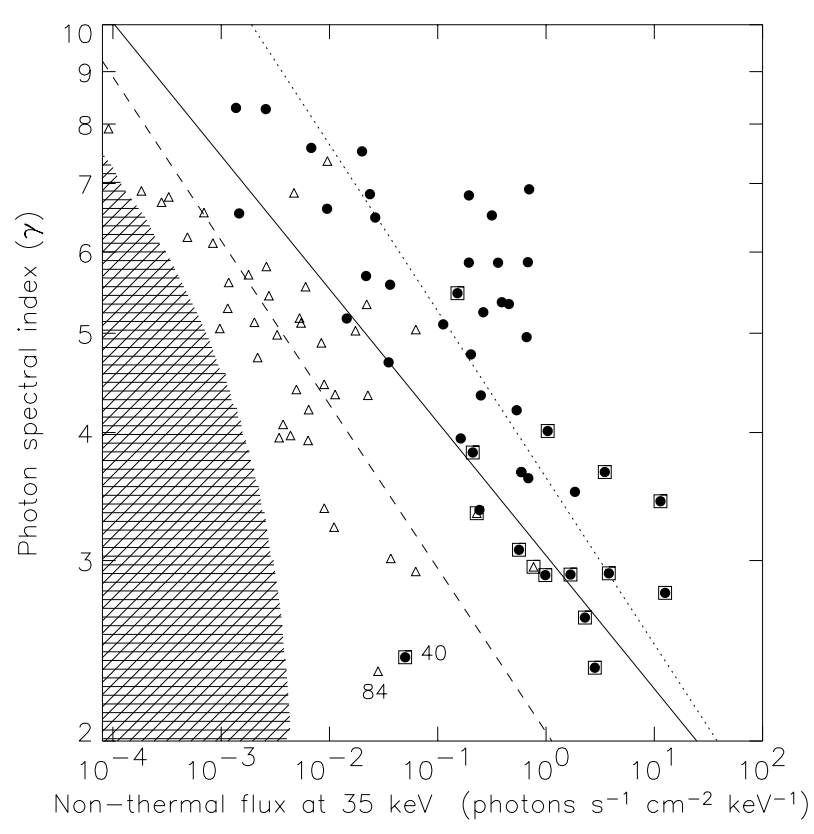

Fig. 2. Spectral index $\gamma$ versus RHESSI non-thermal photon flux at $35 \mathrm{keV}, F_{35}$. The solid line represents the linear bisector regression, given in Eq. (4). Flares with GOES class smaller than $\mathrm{C} 2$ are indicated with a triangle, those larger than $\mathrm{C} 2$ with a circle. The points outlined by a square are the events which where fitted with a broken power-law. The dotted line represents the regression for events larger than $\mathrm{C} 2$, and the dashed line represents the regression for events smaller than C2. Events in the shaded region would not appear in the flare list and would not be selected. The numbers refer to the event list in Table 1. These events are discussed in Sect. 3.4.

single best regression model. The ordinary least squares regressions of $y$ vs. $x$ and $x$ vs. $y$ yield a limit for the confidence range of $\alpha=0.13 \pm 0.07$.

As discussed in Sect. 2.4 there was more than one possible fitting model for most of the events smaller than C2 (after background subtraction). Therefore, the set has been divided into events smaller and larger than $\mathrm{C} 2$, and an independent regression has been made for both parts. The non-thermal flux of both sets ranges over 4 orders of magnitude and their spectral index ranges from 2.3 up to 8.3. The regression of the low GOES-class flares leads to a relation

$\gamma=2.04 F_{35}^{-0.16}$

with confidence range $0.16 \pm 0.05$ for the exponent. These flares are indicated with a triangle in Fig. 2. When taking only the events larger than $\mathrm{C} 2$, the relation becomes

$\gamma=3.60 F_{35}^{-0.16}$,

with confidence range $0.16 \pm 0.06$ for the exponent. Both sets of flares thus yield the same slope in logarithmic $F_{35}$ vs. $\gamma$ dependence. For the flares larger than $\mathrm{C} 2$ however, the regression line is shifted to higher $F_{35}$ values by about one order of magnitude (compare Fig. 2). This leads to a flatter slope, when the regression is performed for the whole data set.

We are interested in the difference between small and large flares and whether their non-thermal parameters have something in common with the parameters of several sub-peaks of 


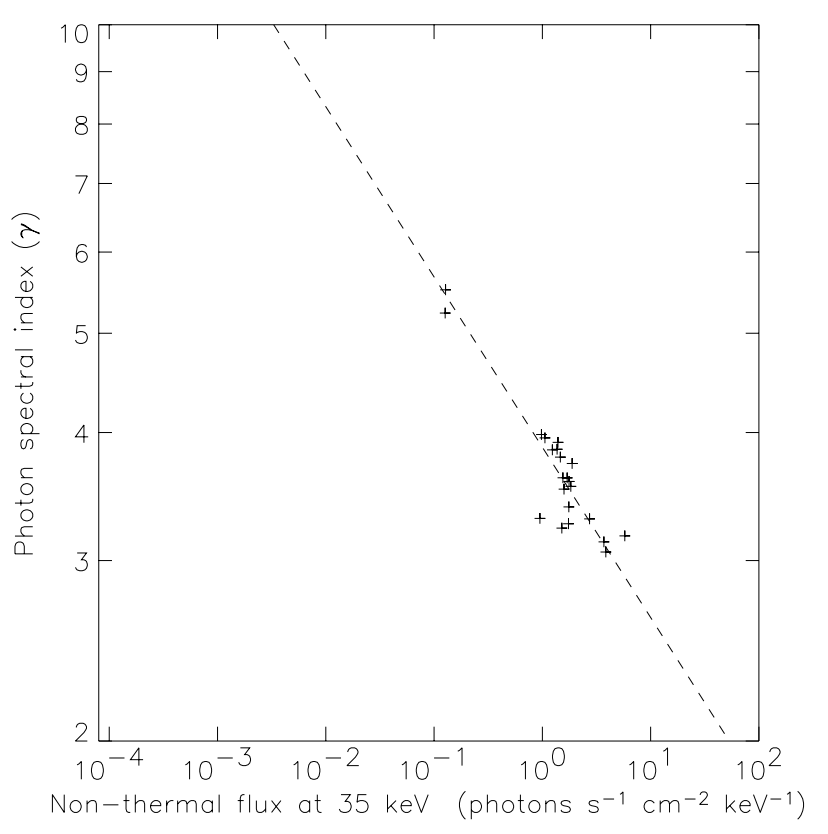

Fig. 3. Spectral index $(\gamma)$ versus non-thermal photon flux at $35 \mathrm{keV}$ for several sub-peaks of a single flare. The dashed line represents the regression line (Eq. (7)).

one single flare. Therefore, the same plot and regression has been made for sub-peaks of one of the flares that have been analyzed by GB04 (event No. 23 in their list, not present in our list). This plot is shown in Fig. 3. The relation is

$\gamma=3.89 F_{35}^{-0.17}$,

and the confidence range for the exponent $\alpha=0.17 \pm 0.01$. The relation between $\gamma$ and $F_{35}$ is quite similar for several peaks of one flare as for many flares of different size at time of maximum non-thermal emission. Note that the maximum non-thermal sub-peak flux of the inspected flare (GOES M4.9) ranges from $10^{-1}$ to $10^{1}$ photons $\mathrm{s}^{-1} \mathrm{~cm}^{-2} \mathrm{keV}^{-1}$ and therefore represents only a small section of the flux range of all flares in the presented sample. The observed power-law component (Eq. (7)) is, however, very close to the relation for only events with GOES class larger than $\mathrm{C} 2$.

\subsubsection{Observational limits}

As mentioned in Sect. 2.1.1 there is a selection effect due to the nature of the flare list (only events with emission above background in the $12-25 \mathrm{keV}$ band are listed). We made an estimate on how the $\gamma$ vs. $F_{35}$ relation is influenced by this selection effect.

The idea is to simulate count spectra and find limits for $F_{35}$ and $\gamma$ for which the total number of counts in the $12-25 \mathrm{keV}$ energy range is larger than $3 \sigma$ of the background. The spectral response matrix (SRM) without attenuators has been used. The average background in counts $\mathrm{s}^{-1}$ in the $12-25 \mathrm{keV}$ energy band was estimated from the light curve. Photon spectra have been calculated, assuming a thermal component and an unbroken power-law. The temperature and emission measure were assumed according to the relations given in Eqs. (9) and (10). The low energy turnover was fixed at $9 \mathrm{keV}$. The photon spectra have been calculated for several values of spectral index $\gamma$ from the interval $[2,10]$ for a number of non-thermal fluxes $F_{35}$ from the interval $\left[10^{-5}, 10^{-2}\right]$ photons s${ }^{-1} \mathrm{~cm}^{-2} \mathrm{keV}^{-1}$. Each of these photon spectra has been multiplied by the SRM to get count spectra. For each flux $F_{35}$ and spectral index $\gamma$ in the above given intervals, the total number of counts in the energy range $12-25 \mathrm{keV}$ has been calculated and compared to the background counts. For every $F_{35}$ there exists a limit in $\gamma$ where the total simulated counts are less than $3 \sigma$ of the background counts. These boundaries plotted in the $\gamma$ vs. $F_{35}$ relation define a region (shaded region in Fig. 2) where events do not appear in the flare list.

From the location of the observational limits and the data points on the plot, one can expect the selection effect to be largest for soft events with a small non-thermal flux. If such events are missing in our selection, the regression line would be flatter in reality.

\subsubsection{Energy of "equal photon flux"?}

As weaker flares are softer, there must be a region where spectra of weak and strong flares intersect. A "pivot region" is sometimes found for the SHS evolution of single subpeaks (see Introduction). Similarly, an energy $E_{\mathrm{eq}}$ or an energy range may exist where small and large flares have equal non-thermal photon flux $F_{\text {eq. }}$.

We have searched for an "energy of equal photon flux" by calculating the energy distribution of all intersections between the non-thermal power-law fittings. The half width is extremely broad, ranging from 2.1 to $52 \mathrm{keV}$ with a peak at $10.4 \mathrm{keV}$.

\subsubsection{High-energy break}

As mentioned before, 15 spectra have been fitted with a highenergy break. These events are indicated with a square in Fig. 2. The non-thermal flux $F_{35}$ of all these events is higher than $4 \times$ $10^{-2}$ photons $\mathrm{s}^{-1} \mathrm{~cm}^{-2} \mathrm{keV}^{-1}$. Thus high-energy breaks are observed only in large flares.

The break energy does not correlate with the spectral index. However a correlation appears between the spectral index below break energy $(\gamma)$ and the spectral index above break en$\operatorname{ergy}(\beta)$ following a relation $\beta=(1.96 \pm 0.20) \gamma-1.59$. A similar relation has previously been reported by Lin \& Schwartz (1986). Further, we observed a correlation between the break energy and the non-thermal photon flux at $35 \mathrm{keV}$, indicating that flares with smaller non-thermal flux have on the average a lower break energy. Such a comparison has previously been made by Dulk et al. (1992), who found no correlation between break energy and non-thermal flux.

However, we were not able to exclude without doubt selection effects that could lead to these correlations (see also discussion in Sect. 4). 


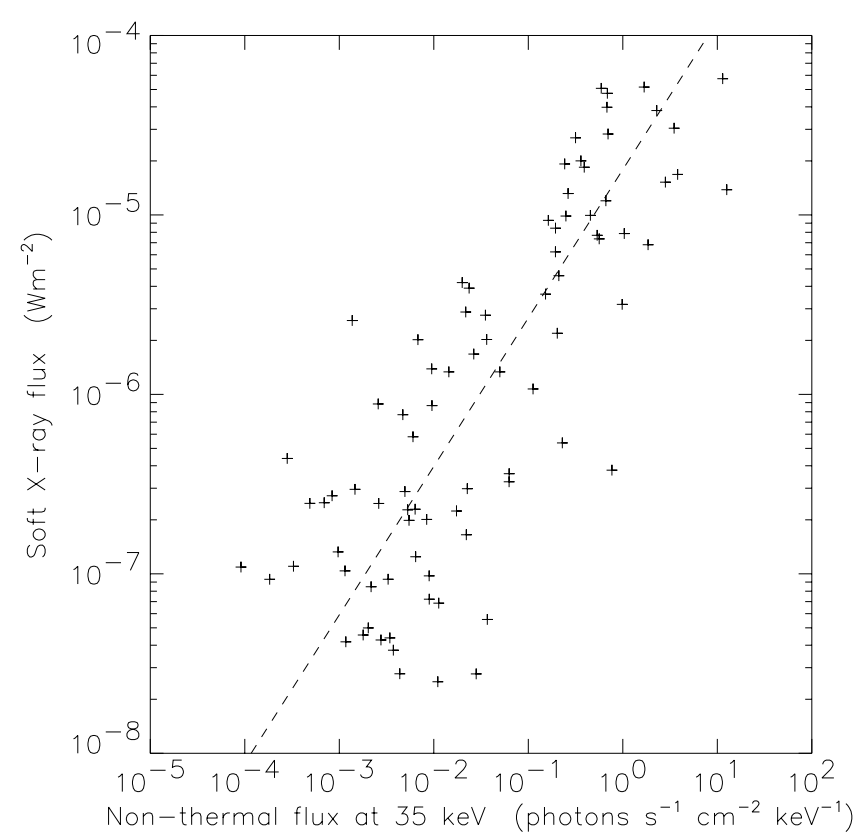

Fig. 4. Soft X-ray flux $F_{\mathrm{G}}$ versus non-thermal flux $F_{35}$ at $35 \mathrm{keV}$ with regression line, as given in Eq. (8).

\subsection{Thermal flare properties compared to non-thermal properties}

In a next step the soft X-ray flux observed by the GOES satellites was included in the analysis. The GOES flux of all flares at time of maximum emission (GOES peak time) in the 1-8 band has been extracted. For a proper analysis, the background was subtracted. The background was chosen in GOES light curves as either linearly interpolated between pre-flare and post-flare background or as constant. Subtracting background changes the GOES class contrary to the usual classification. When referring to the size of a flare, the GOES class according to peak flux without background subtraction is given throughout this work. For comparing physical parameters, however, the background-subtracted values have been used.

First, the non-thermal flux $F_{35}$ has been compared to the maximum GOES flux, $F_{\mathrm{G}}$. There is a linear correlation in a double logarithmic plot, presented in Fig. 4, giving:

$F_{\mathrm{G}}=1.8 \times 10^{-5} F_{35}^{0.83}$

where the exponent $b=0.83 \pm 0.19 . F_{\mathrm{G}}$ is in units of $\mathrm{W} \mathrm{m}^{-2}$ and $F_{35}$ in photons $\mathrm{s}^{-1} \mathrm{~cm}^{-2} \mathrm{keV}^{-1}$.

As $F_{35}$ vs. $\gamma$ and $F_{35}$ vs. $F_{\mathrm{G}}$ correlate, one must expect a correlation between $F_{\mathrm{G}}$ and $\gamma$. Figure 5 illustrates that this is not the case. From the relations $\gamma=A F_{35}^{\alpha}$ and $F_{\mathrm{G}}=C F_{35}^{d}$ one can calculate an expected relation $\gamma=A C^{-\alpha / d} F_{\mathrm{G}}^{\alpha / d}$, giving $\gamma=0.53 F_{\mathrm{G}}^{-0.16}$, represented by the dashed line. As expected, the flares with small $F_{35}$ are in the upper left corner and the ones with large $F_{35}$ in the bottom right. However, the scatter in $\gamma$ is so large that the correlation is statistically not significant.

We compared also the temperature $T_{1}$ and emission measure $E M_{1}$ of the thermal plasma derived from RHESSI data, to the non-thermal X-ray flux at $35 \mathrm{keV}$. Figure 6 indicates

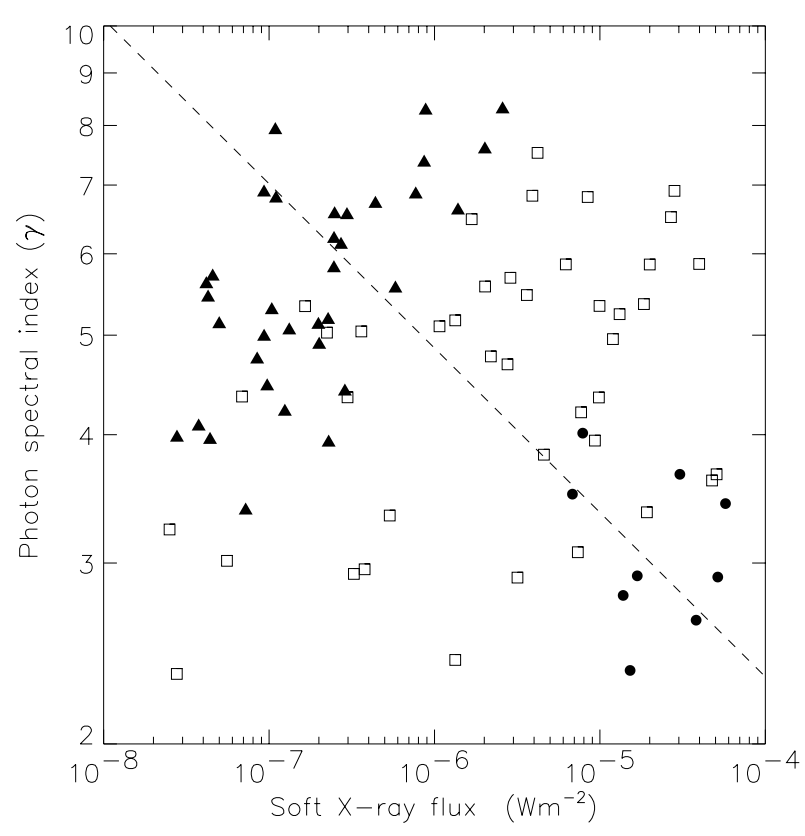

Fig. 5. Spectral index $\gamma$ versus soft X-ray flux $F_{\mathrm{G}}$. The data points have been labeled with 3 different symbols according to 3 flux intervals in $F_{35}$ (units: photons $\mathrm{s}^{-1} \mathrm{~cm}^{-2} \mathrm{keV}^{-1}$ ). Triangles: $F_{35}$ in the interval $\left[10^{-5}, 10^{-2}\right]$; squares: $F_{35}$ in the interval $\left[10^{-2}, 10^{0}\right]$; circles: $F_{35}$ in the interval $\left[10^{0}, 10^{2}\right]$. The dashed line is the expected trend-line from the correlations of $\gamma$ vs. $F_{35}$, and $F_{\mathrm{G}}$ vs. $F_{35}$.

a correlation between temperature and non-thermal flux with a relation

$T_{1}=1.46 \ln F_{35}+21.57$,

where $T_{1}$ is in units of $10^{6} \mathrm{~K}$ and $F_{35}$ in photons s $\mathrm{cm}^{-1} \mathrm{keV}^{-1}$. A higher non-thermal flux is therefore associated with a higher temperature. A similar observation holds for the relation between $F_{35}$ and the emission measure (Fig. 7).

$E M_{1}=5 \times 10^{48} F_{35}^{0.91}$,

with $E M_{1}$ in $\mathrm{cm}^{-3}$.

\subsection{Thermal flare plasma}

Similar to the investigations by Feldman et al. (1996), we also compared the temperature $T_{1}$ at hard X-ray peak time to the maximum soft X-ray flux $F_{\mathrm{G}}$. We note a correlation in Fig. 8, giving

$F_{\mathrm{G}}=3.5 \times 10^{0.33 T_{1}-12}$,

where $F_{\mathrm{G}}$ is in $\mathrm{W} \mathrm{m}^{-2}$, plotted in a logarithmic scale, and $T_{1}$ is in units of $10^{6} \mathrm{~K}$, plotted in linear scale. Due to the large scatter, the power factor $\mathrm{p}$ in Eq. (11) has a confidence range of $p=0.33 \pm 0.29$. The Feldman et al. result $(F(T)=3.5 \times$ $10^{0.185 T-9.0}$ ) differs considerably, as is illustrated in Fig. 8. It is though still within the estimated range for the regression parameters. Contrary to Feldman et al., we used background subtracted GOES flux. The background-subtraction from the GOES data has the biggest influence on the smaller flares, altering the flux to smaller values and extending the flux range one order of magnitude. Using the original, not background subtracted GOES data, we get $F_{\mathrm{G}}=1.7 \times 10^{0.25 T_{1}-10}$. 


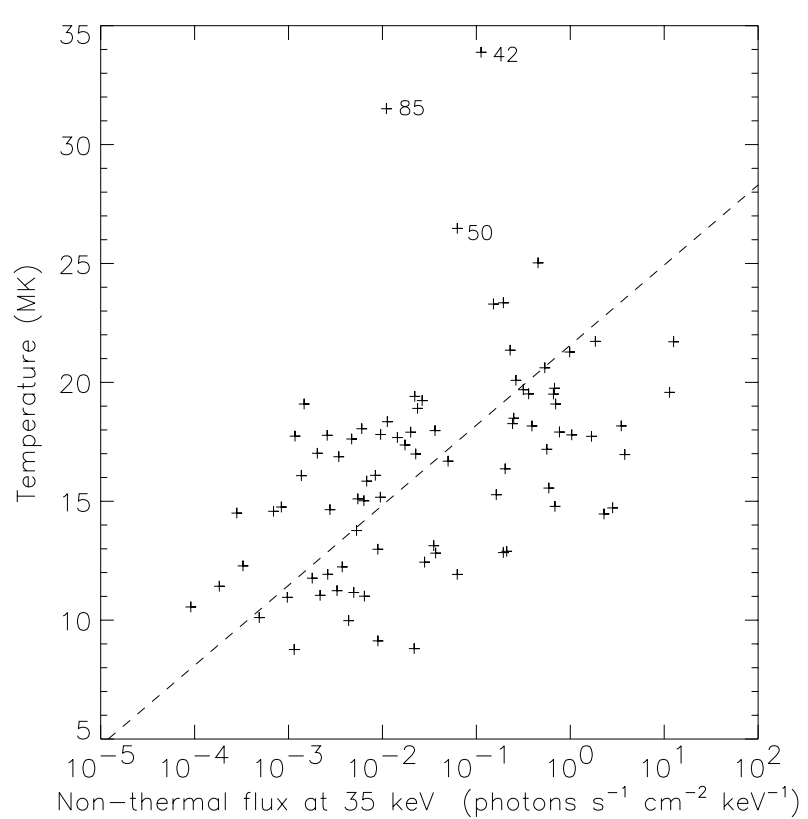

Fig. 6. RHESSI temperature $T_{1}$ versus non-thermal flux at $35 \mathrm{keV}$ with linear bisector regression line (Eq. (9)). The numbers refer to the event list in Table 1.

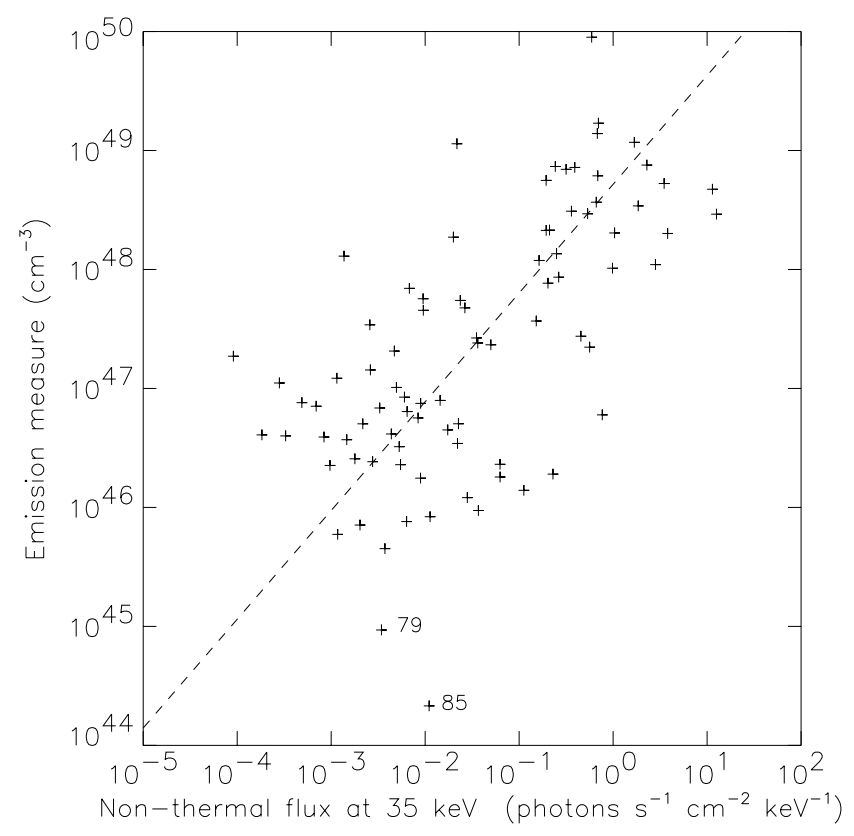

Fig. 7. Emission measure $E M_{1}$ versus non-thermal flux at $35 \mathrm{keV}$. The dashed line indicates the linear bisector regression line (Eq. (10)). The numbers refer to the event list in Table 1.

\subsubsection{Comparison of temperatures}

From the flux ratio in the 2 GOES wavelength bands, one can calculate an isothermal temperature $T_{\mathrm{G}}$ at the time of maximum soft X-ray flux, using Mewe spectral models (Mewe et al. 1985). As the minimum energies of the two bands are $1.5 \mathrm{keV}$ and $3.1 \mathrm{keV}$ respectively, GOES measurements are dominated by photons at lower energy than used to determine the temperature from RHESSI data $(\geq 4 \mathrm{keV})$. A comparison of the two measurements is also a check on the applied methods: flux

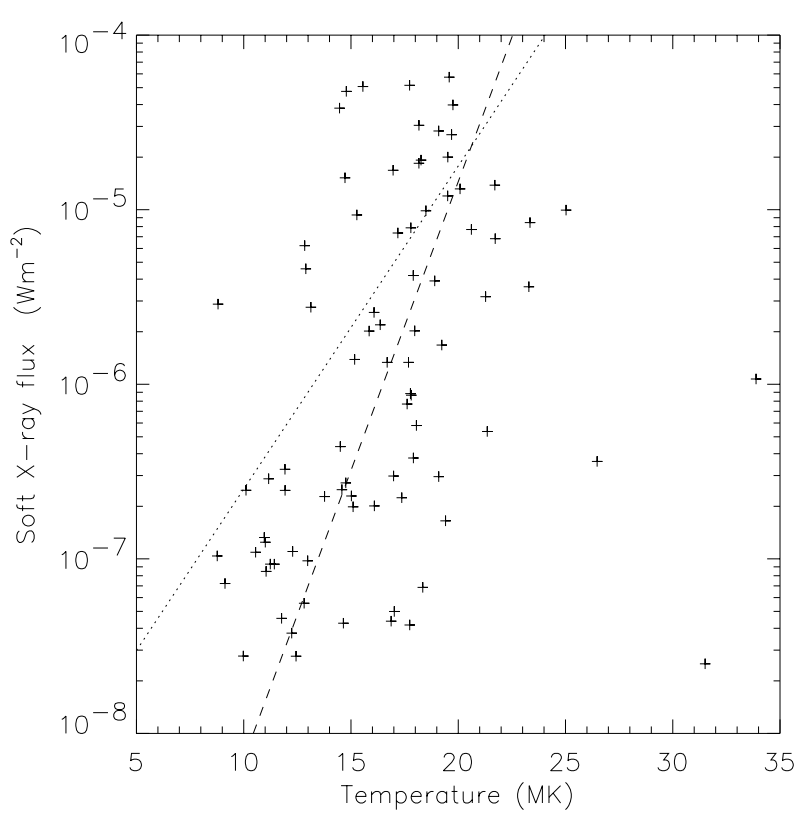

Fig. 8. Maximum soft X-ray flux versus temperature. The dashed line gives the linear bisector regression to the data (Eq. (11)). The dotted line is the trend line calculated from the Feldman et al. result (Eq. (1)).

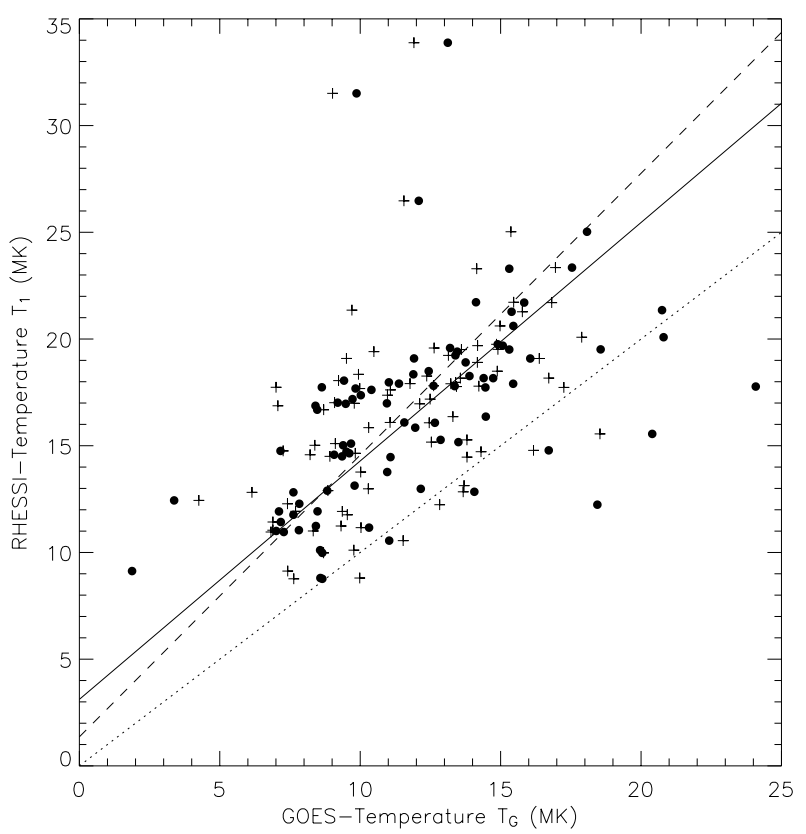

Fig. 9. Temperature measured by RHESSI $\left(T_{1}\right)$ compared to GOES temperature $\left(T_{\mathrm{G}}\right)$. The dotted line indicates the diagonal line. Crosses indicate the GOES temperature at GOES peak time, the dashed line gives the corresponding regression line. Dots give the GOES temperature measured at the same time as the RHESSI temperature with the solid line as regression line.

ratio (GOES, at soft X-ray peak time) versus spectral fitting (RHESSI, at hard X-ray peak time). Figure 9 indicates that the temperature derived from RHESSI data is often higher. A linear bisector regression yields a relation $T_{1}=1.31 T_{\mathrm{G}}+1.47$ (in MK), but is influenced by the three outliers. Without the outliers, the relation would be $T_{1}=1.12 T_{\mathrm{G}}+3.12$. 
The emission measure derived from the GOES data is higher than that from the RHESSI data for 82 out of 85 events. The ratio $E M_{1} / E M_{\mathrm{G}}$ increases for larger events. On the average, $E M_{1} \approx 0.1 E M_{\mathrm{G}}$. Most likely, the RHESSI measurements emphasize the hotter part of a non-isothermal temperature distribution in the soft X-ray source. Part of these trends could also be accounted for by the different times of measurements, as the hard X-ray peak usually occurs earlier in the course of a flare than the soft X-ray peak. Taking the GOES temperature at the same time as the RHESSI temperature leads indeed to a relation with less pronounced trend: $T_{1}=1.13 T_{\mathrm{G}}+3.17$, but the RHESSI temperature is still higher than the GOES temperature in most cases (Fig. 9).

\subsection{Inspection of exceptional flares}

In most of the presented plots there are a few events far from the overall trend line. The most striking ones have been numbered according to Table 1 and are shortly discussed here.

Nos. 40 and 84 have very hard spectral indices, No. 84 has the hardest $\gamma$ in our data set. Its spectrogram shows short, impulsive emission up to over $30 \mathrm{keV}$.

Nos. 42, 50 and 85 have very high temperatures. The spectrograms of all three events indicate a very short, impulsive, non-thermal peak with emission over $30 \mathrm{keV}$.

No. 79 has a small emission measure of only $9.3 \times 10^{44} \mathrm{~cm}^{-3}$. The temperature of $16.9 \mathrm{MK}$ is rather high for a flare of this size. Again, the flare is very short and impulsive.

The common characteristic of all these flares is the short duration of the non-thermal emission.

\section{Discussion}

The high spectral resolution of RHESSI's germanium detectors permits the separation of a non-thermal and one or two thermal components in most X-ray spectra of solar flares over a large range of flare sizes. The selection was made according to the usual definition of flare size in terms of peak soft X-ray flux (labeled B1 to M6) as measured by the GOES $1-8 \AA$ A channel. It remains nearly uniform in soft X-ray peak flux after background subtraction but extends one more order of magnitude to smaller flares (A1 to M6).

The analysis of the non-thermal emission shows a clear relation between the spectral index $\gamma$ and the power-law normalization $F_{35}$ at peak time. The correlation between $\gamma$ and $F_{35}$ can be expressed by a power-law. We note that the quantitative relation is not identical to the relation found for the SHS behavior in subpeaks of events. The spectral index $\gamma$ depends less on $F_{35}$, and the power-law exponent $\alpha$ is smaller (Eq. (4)). The two exponents $\alpha$ for the temporal evolution of single flares and the peak values of many flares can be brought closer together when we accept that the flares smaller in soft X-rays (and GOES class) are generally deficient in $F_{35}$, relative to the $F_{35}$ vs. $\gamma$ relation found by GB04. Soft events with small hard
X-ray flux are missing by selection. This could lead to a flatter relation in reality.

Further, it may well be possible that the power-law relation does not hold below $F_{35} \approx 10^{-5}$ photons s $\mathrm{s}^{-1} \mathrm{~cm}^{-2} \mathrm{keV}^{-1}$, but flattens toward a constant value of $\gamma$.

The selection according to thermal flare properties aims also at a large range of non-thermal $\mathrm{X}$-ray fluxes. They range from $10^{-4}$ to 10 photons s${ }^{-1} \mathrm{~cm}^{-2} \mathrm{keV}^{-1}$ at $35 \mathrm{keV}\left(F_{35}\right.$, see Fig. 2), with a relatively uniform distribution between $10^{-3}$ and 1 photons $\mathrm{s}^{-1} \mathrm{~cm}^{-2} \mathrm{keV}^{-1}$. The non-thermal flux distribution depends on the reference energy. At lower reference energy, in the range of the "equal photon flux", the distribution becomes narrower.

A selection effect comes in when the comparison includes parameters of the thermal plasma, since among small flares events with high non-thermal flux have been preferentially selected. The relations shown in Figs. 4 and 5 may be affected by selection. In Fig. 4 small soft X-ray events with low $F_{35}$ are missing, particularly in the interval $\left[10^{-5}, 10^{-3}\right] F_{35}$ and $\left[10^{-8}, 10^{-7}\right] F_{\mathrm{G}}$. Thus the regression line may be slightly flatter and the exponent in Eq. (8) smaller. The trend for larger scatter in $F_{35}$ for smaller flares may thus be even larger than visible in Fig. 4. In Fig. 5 small soft X-ray events with large $\gamma$ (upper left corner) are missing. This may contribute to the absence of significant correlation between $\gamma$ and soft X-ray flux. Note, however, that the three intervals of non-thermal flux line up along the expected correlation line with a large scatter below and above the line. Nevertheless, it remains a fact that one cannot predict the spectral index at peak non-thermal emission from the soft X-ray flux.

Different selection effects have already been mentioned in relation with the break energy of the non-thermal spectrum at high energies, visible only in large flares (high $F_{35}$ ) and low $\gamma$. We further note that using the spectral index above the break energy, $\beta$ and its corresponding flux normalization at $35 \mathrm{keV}$ worsens the correlation.

It may be added here also that the low-energy turnover $E_{\text {turn }}$ seemingly correlates with $F_{35}$ and even better with $F_{\mathrm{G}}$. The latter and the fact that $E_{\text {turn }}$ is usually close to the energy with equal thermal and non-thermal contributions strongly point to a spurious effect (see also Saint-Hilaire \& Benz 2005). It does not influence significantly the relations discussed above.

The temperature changes only slightly (from about $5 \mathrm{MK}$ to $30 \mathrm{MK}$ ) over 4 mag of $F_{\mathrm{GOES}}$ but there is a linear dependence between $F_{\mathrm{GOES}}$ and $E M_{1}$. Therefore, the similarity of Figs. 5 and 8 is not surprising. In fact, the two exponents in Eqs. (8) and (10) are identical within the statistical uncertainty. However, it is surprising that the correlation does not improve from $F_{\mathrm{G}}$ vs. $F_{35}$ to $E M_{1}$ vs. $F_{35}$, as $E M_{1}$ is determined simultaneously and at the peak of $F_{35}$ and does not contain the temperature, whereas $F_{\mathrm{G}}$ is determined at the peak of the soft X-ray emission later in the flare. $F_{\mathrm{G}}$ correlates well with $E M_{1}$

$F_{\mathrm{G}}=3.6 \times 10^{-50} E M_{1}^{0.92}$

with $E M_{1}$ in $\mathrm{cm}^{-3}$ and $F_{\mathrm{G}}$ in $\mathrm{W} \mathrm{m}^{-2}$, and $b=0.92 \pm 0.09$. The exponent $b$ is not significantly below unity. Such a trend may arise from the reduced efficiency for thermal emission at high 
temperatures of large flares, or may be due to larger energy losses because of longer duration.

The correlation between the temperature and soft X-ray emission of the thermal plasma (Fig. 8) is steeper than previously reported by Feldman et al. (1996 see Eq. (1)). Part of the difference can be accounted for by our subtraction of the background, increasing the range of peak fluxes. On the other hand, Feldman et al. used temperatures from the Yohkoh BCS detectors. These detectors saturate at high fluxes. Therefore the correlation found by Feldman et al. may be even flatter. As already mentioned in Sect. 3.3.1 RHESSI might measure the hotter part of a non-isothermal plasma, whereas GOES sees a cooler part.

Finally, none of the investigations on the flare position on the disk showed a significant effect. The distributions of $\gamma, F_{35}$, and $E_{\mathrm{br}}$ are independent of radial distance from the center of the disk. Events with position offset larger than 950 arcsec from the sun center have been looked at separately. They show no significant pattern on the $\gamma$ vs. $F_{35}$ plot. This excludes an influence of the varying albedo of the X-ray emission scattered by the chromosphere on the above results.

\section{Summary and conclusions}

Flares observed by RHESSI were selected from a wide range of GOES flare size. The selection was nearly uniform from B1 to M6 (corresponding to flares between A1 to M6 after background subtraction). Out of 92 flares with a well defined spectrum at peak time, 85 events can be fitted by a thermal and a non-thermal component. Only 6 events (7\%) had only thermal emission, one had only a non-thermal component. The fitted flux at $35 \mathrm{keV}$ at the peak time of the non-thermal emission in 85 flares ranges over more than 5 orders of magnitude (Fig. 2). This greatly exceeds the range that has been analyzed in the temporal SHS evolution of a single subpeak (Fig. 3) and even in all combined subpeaks in the literature (GB04). A powerlaw index could be reliably determined from a minimum value of 2.3 up to a maximum of 8.3 .

The main conclusion based on this study is that the spectral index $\gamma$ at peak non-thermal emission of flares, as well as at subpeaks of a single flare correlate. Thus flares with small non-thermal flux are softer on the average. This makes them even more difficult to detect in high-energy X-ray emission. Eventually, the non-thermal emission becomes indistinguishable from thermal emission, although it represents possibly a larger amount of energy.

We have studied further the relations between non-thermal flux, spectral index, soft X-ray flux, temperature, and emission measure with the following results:

- The spectral index, $\gamma$, and the peak non-thermal flux at $35 \mathrm{keV}, F_{35}$, correlate linearly in a double logarithmic plot, similar to the time evolution of $\gamma$ and $F_{35}$ during one single flare or subpeak.

- Small soft X-ray emitting flares have a lower $F_{35}$ by nearly an order of magnitude from the relation derived earlier. They also show a larger spread in $F_{35}$.
- Soft X-ray flux and non-thermal flux at $35 \mathrm{keV}$ are correlated, indicating that flares with larger non-thermal emission have larger thermal emission.

- Although soft X-ray flux and non-thermal flux are correlated, as well as spectral index and non-thermal flux, there is no significant correlation between spectral index and soft $\mathrm{X}$-ray flux (GOES class). Probable reasons are the large scatter in $\gamma$ and a selection effect.

- Temperature and emission measure of the thermal plasma both correlate with non-thermal flux, indicating that flares with large non-thermal flux have a higher temperature and emission measure.

- A comparison of soft X-ray flux and temperature yields a correlation also seen in previous work, but with slightly different relation due partially to background subtraction.

That the temperature is higher in large flares is the property of the thermal flare plasma, thought to be heated by precipitating particles, thus a secondary product of energy release. It may be explained by the repeated impact of electrons accelerated in elementary flares, the larger the flare, the more such impacts and the hotter the target. The $\gamma$ vs. $F_{35}$ relation, however, concerns the non-thermal electron population thought to be a primary flare product. As it applies to different flares and not only to the temporal evolution in a flare, it cannot be a secondary phenomenon. Thus it is an intrinsic feature of the acceleration process. Its quantitative relation must be accounted for by a realistic acceleration theory.

Lower $F_{35}$ being associated with larger $\gamma$ affect the frequency distribution of flare energies. If the energy or peak flux of non-thermal electrons is determined well above the mean energy of "equal photon flux" $(10.4 \mathrm{keV})$, flares with low nonthermal flux are lost in the background. For RHESSI observations, this affects the selection for GOES classes smaller than about C2 (Fig. 2). The frequencies of hard X-ray flares are reported to have power-law distributions (e.g. Hudson 1991). If soft small flares have been missed, the published power-law indices must be considered as lower limits.

Acknowledgements. RHESSI data analysis at ETH Zürich is supported by ETH grant TH-1/04-2 and the Swiss National Science Foundation (grant 20-105366). Much of this work relied on the RHESSI Experimental Data Center (HEDC) developed under ETH Zürich grant TH-W1/99-2. We thank the many people who have contributed to the successful operation of RHESSI and acknowledge P. Saint-Hilaire for providing the list of events with a good image and helpful discussions.

\section{References}

Benz, A. O., \& Grigis, P. C. 2002, Sol. Phys., 210, 431

Benz, A. O., Hubert, P., Saint-Hilaire, P., \& Zlobec, P. 2005, Adv. Space Res., in press

Dougherty, B. L., Zirin, H., \& Hsu, K. 2002, ApJ, 577, 457

Dulk, G. A., Kiplinger, A. L., \& Winglee, R. M. 1992, ApJ, 389, 756 
Feldman, U., Doschek, G. A., Behring, W. E., \& Phillips, K. J. H. Matthews, S. A., van Driel-Gesztelyi, L., Hudson, H. S., \& Nitta, N. V. 1996, ApJ, 460, 1034

Garcia, H. A. 1994, Sol. Phys., 154, 275

Grigis, P. C., \& Benz, A. O. 2004, A\&A, 426, 1093

Hudson, H. S. 1991, Sol. Phys., 133, 357 2003, A\&A, 409, 1107

Mewe, R, Gronenschild, E. H. B. M., \& van den Oord, G. H. J. 1985, A\&AS, 62, 197

Parks, G. K., \& Winckler, J. R. 1969, ApJ, 155, 117

Isobe, T., Feigelson, E. D., Akritas, M. G., \& Babu, G. J. 1990, ApJ, 364, 104

Kahler, S. W. 1982a, J. Geophys. Res., 87, 3439

Kahler, S. W. 1982b, ApJ, 261, 710

Krucker, S., Christe, S., Lin, R. P., Hurford, G. J., \& Schwartz, R. A. 2002, Sol. Phys., 210, 445

Lin, R. P., \& Schwartz, R. A. 1986, ApJ, 312, 462

Lin, R. P., Dennis, B. R., Hurford, G. J., et al. 2002, Sol. Phys., 210, 3

Lu, E. T., \& Hamilton, R. J. 1991, ApJ, 380, L89

Saint-Hilaire, P., Von Praun, C., Stolte, E., et al. 2002, Sol. Phys., 210, 143

Saint-Hilaire, P., \& Benz, A. O. 2005, A\&A, 435, 743

Schwartz, R. A. 1996, Compton Gamma Ray Observatory Phase 4 Guest Investigator Program: Solar Flare Hard X-ray Spectroscopy, Technical Report, NASA Goddard Space Flight Center

Lu, E. T., Hamilton, R. J., McTiernan, J. M., \& Bromund, K. R. 1993, ApJ, 412, 841

Shibata, K., \& Yokoyama, T. 1999, ApJ, 526, L49

Simnett, G. M., \& Benz, A. O. 1986, A\&A, 165, 227

Smith, D. M., Lin, R. P., Turin, P., et al. 2002, Sol. Phys., 210, 33 